\title{
ON UNIFORM APPROXIMATION BY RATIONAL FUNCTIONS ${ }^{1}$
}

\section{LYNN A. STEEN}

If $X$ is a compact subset of the complex plane, $C(X)$ will denote the sup norm algebra of continuous complex-valued functions on $X$; $R(X)$ will denote the uniformly closed subalgebra of $C(X)$ generated by the rational functions with poles off $X$.

Whenever $X$ has an interior, $R(X)$ is a proper subalgebra of $C(X)$. Mergelyan [1] has shown that it is still possible for $R(X)$ to be strictly contained in $C(X)$ even if $X$ has no interior: $R(X) \neq C(X)$ whenever $X$ is a set formed by deleting from the interior of the closed unit disc a sequence of open discs with mutually nonintersecting boundaries of finite total length in such a way as to leave no interior.

In order to understand how this $R(X)$ could be a proper subalgebra of $C(X)$, it had been conjectured that any such $R(X)$ must be antisymmetric, i.e., must contain no nonconstant real-valued function. This conjecture will be proved false by the construction of a counterexample.

The strategy of construction is to mark off on the horizontal diameter of the unit disc a general Cantor set. The vertical lines determined by the endpoints of the intervals complementary to the Cantor set then serve as guidelines which will be covered almost everywhere by open discs to be deleted from the unit disc. In order to insure that the total length of the circumferences of the deleted discs is finite, it is necessary to alternate between selecting an interval complementary to the Cantor set, and selecting the discs which cover the associated pair of guidelines: at each stage we must select the interval in such a way (i.e., large enough) as to insure that a large portion of the length of the associated guidelines is already deleted by the discs which were selected at the preceding stages.

The final step-that of showing that the algebra $R(X)$ is not antisymmetric-involves proving that the Cantor function (extended as a constant in the vertical direction) is in $R(X)$. This proof relies on a lemma due to Mergelyan [1], which may be stated in this form:

Received by the editors January 5, 1966.

1 The research in this paper forms part of the author's doctoral dissertation, submitted to the Massachusetts Institute of Technology in August, 1965. The author is indebted to Professor Kenneth Hoffman for guidance and ideas in the course of this research. 
Lemma. Let $X$ be the set formed by deleting from the closed unit disc $D$ certain pairwise disjoint open subdiscs $D_{1}, D_{2}, \cdots, D_{n}$, centered at points $\lambda_{1}, \lambda_{2}, \cdots, \lambda_{n}$, of radii $r_{1} \geqq r_{2} \geqq \cdots \geqq r_{n}>0$. Let $f \in C(D)$ and let $Z$ denote the set of interior points of $X$ at which $f$ is not analytic. If $\delta \geqq \sup \{d(z, \partial X) \mid z \in Z\}$ and if $\delta \geqq r_{n}$, then there exists a rational function $r$ with poles at $\lambda_{1}, \lambda_{2}, \cdots, \lambda_{n}$, such that

$$
\|f-r\|_{x} \leqq c \cdot \omega(f ; \delta) \cdot\left(\delta / r_{n}\right)^{2}
$$

where $c$ is an absolute constant, and $\omega(f ; \delta)$ is the modulus of continuity of $f$ on $D$, defined by

$$
\omega(f ; \delta)=\sup _{x, y \in D ;|x-y| \leqq \delta}|f(x)-f(y)| .
$$

To begin construction of the counterexample, we will define on $[-1,1]$, the horizontal diameter of the unit disc, a general Cantor set by the usual process: at stage $n-1$ we remove one open interval from each of the $2^{n-2}$ closed intervals which resulted from stage $n-2$ -leaving $2^{n-1}$ closed intervals. We denote the $2^{n}$ endpoints of these $2^{n-1}$ intervals by $E_{n}=\left\{s_{n}^{k}\right\}_{k=1}^{2^{n}}$ where $s_{n}^{k}<s_{n}^{k+1}$. Then we must have $E_{n} \subset E_{n+1}$, and in particular,

$$
s_{n}^{2 i+1}=s_{n+1}^{4 i+1}<s_{n+1}^{4 i+2}<s_{n+1}^{4 i+3}<s_{n+1}^{4 i+4}=s_{n}^{2 i+2} .
$$

Corresponding to each $E_{n}$, we define $Y_{n}$ to be a compact subset of the closed unit disc formed by removing the interiors of certain disjoint closed discs centered on the set of guidelines $\hat{E}_{n}=\left\{x+i y \mid x \in E_{n}\right\}$. $Y_{n+1}$ will be constructed from $Y_{n}$ by simply removing certain open discs centered on $\hat{E}_{n+1}-\hat{E}_{n}$. For each positive integer $k$, we define ${ }_{k} Y_{n}$ to be the set formed from $Y_{n}$ by filling in all holes of radius (strictly) less than $3^{-k}$. That is,

$$
{ }_{k} Y_{n}=Y_{n} \cup\left\{\text { all components of } Y_{n}^{\prime} \text { of radius }<3^{-k}\right\} .
$$

We will construct inductively the sets $E_{1}, E_{2}, \cdots, E_{n}, \cdots$, $Y_{1}, Y_{2}, \cdots, Y_{n}, \cdots$, together with a strictly increasing sequence of positive integers $m_{1}<m_{2}<\cdots<m_{n}<\cdots$, such that (if $L$ represents length):

(i) $L\left(\hat{E}_{n} \cap Y_{n}\right)=0$,

(ii) $L\left(\partial Y_{n}\right) \leqq 4 \pi\left(1-2^{-n}\right)$,

(iii) whenever $m=m_{1}, m_{2}, \cdots, m_{n}$, or $m \geqq m_{n}$,

$$
\sup _{n} \cap_{m} Y_{n} d\left(x, \partial_{m} Y_{n}\right) \leqq\left(1-2^{-n}\right) 3^{1-m} .
$$

To begin, we define $Y_{1}$ to be the closed unit disc, $E_{1}$ to be $\{-1,1\}$, 
and $m_{1}=1$. Then $Y_{1}, E_{1}$, and $m_{1}$ satisfy all the relevant conditions.

We assume now, by induction, that the construction has been carried out up to and including $E_{n}, Y_{n}$, and $m_{n}$. In order to define $E_{n+1}$, we need only specify points of the form $s_{n+1}^{4 i+2}$ and $s_{n+1}^{4 i+3}$ : this we do by requiring $s_{n+1}^{4 i+2}$ to be sufficiently near $s_{n+1}^{4 i+1}=s_{n}^{2+1}$ to insure that

(a) $L\left\{z \in Y_{n} \mid \operatorname{Re} z=s_{n+1}^{4 i+2}\right\} \leqq 4^{-n}$,

(b) whenever $\operatorname{Re} z=s_{n+1}^{4 i+2}$ and $z \in{ }_{m} Y_{n}$ for $m=m_{1}, \cdots, m_{n}$, then

$$
d\left(z, \partial_{m} Y_{n}\right)<\left(1-2^{-(n+1)}\right) 3^{1-m} \text {. }
$$

In a similar manner, we require $s_{n+1}^{4 i+3}$ to be near $s_{n+1}^{4 i+4}=s_{n}^{2 i+2}$.

That such points may in fact be found is guaranteed by the induction hypothesis. Condition (i) implies that

$$
L\left\{z \in Y_{n} \mid \operatorname{Re} z=s_{n+1}^{4 i+1}\right\}=0,
$$

while condition (iii) says that whenever $\operatorname{Re} z=s_{n+1}^{4 i+1}$ and $z \in \in_{m} Y_{n}$ for $m=m_{1}, m_{2}, \cdots, m_{n}$, then

$$
d\left(z, \partial_{m} Y_{n}\right) \leqq\left(1-2^{-n}\right) 3^{1-m}<\left(1-2^{-(n+1)}\right) 3^{1-m} .
$$

Since there are only finitely many (in fact, $n+1$ ) conditions and since each alone may be satisfied by selecting $s_{n+1}^{4 i+2}$ sufficiently near $s_{n+1}^{4 i=1}$, we can therefore satisfy all conditions simultaneously.

Now $Y_{n} \cap\left(\hat{E}_{n+1}-\hat{E}_{n}\right)$ consists of a finite number of closed line segments, and $\partial Y_{n} \cap\left(\hat{E}_{n+1}-\hat{E}_{n}\right)$ consists of the endpoints of these line segments. Each such endpoint lies either on the unit circle, or on the circumference of some disc in $Y_{n}^{\prime}$; let $\rho_{n}$ denote the minimum radius of these discs. Suppose $F$ is some line segment in $Y . \cap\left(\hat{E}_{n+1}-\hat{E}_{n}\right)$; let $\gamma_{1}, \gamma_{2}$ denote the circles that determine the endpoints of $F$. Define

$$
\begin{aligned}
\sigma(F) & =\inf _{x \in F} d\left(x, \partial Y_{n}-\gamma_{1}-\gamma_{2}\right) \\
\sigma_{n} & =\min _{F} \sigma(F) .
\end{aligned}
$$

Clearly $\sigma(F)>0$ for each segment $F$, and since there are only finitely many such segments, $\sigma_{n}>0$. We can thus select $m_{n+1}$ to be the smallest integer such that

(a) $m_{n+1}>m_{n}$,

(b) $3^{-m_{n+1}}<\rho_{n}$,

(c) $3^{-m_{n+1}}<\sigma_{n}$.

Consider now a segment $F$ of $Y_{n} \cap\left(\hat{E}_{n+1}-\hat{E}_{n}\right)$, with bounding circles $\gamma_{1}, \gamma_{2}$. We will remove from $Y_{n}$ the in teriors of pairwise disjoint closed discs centered on $F$, as follows. We first remove sufficiently 
many discs (possibly none) of radius $3^{-m_{n+1}}$ to insure that each remaining point of $F$ is within a distance of $3^{-m_{n+1}}$ of some circle which intersects $F$. (This can always be done, since condition (c) in the definition of $m_{n+1}$ guarantees that if any remaining point $z$ of $F$ should be separated by at least $3^{-m_{n+1}}$ from every circle which intersects $F$, then we can delete an open disc of radius $3^{-m_{n+1}}$ centered at $z$ without intersecting any previously defined holes.) This procedure is then repeated for discs of each radius $3^{-\left(m_{n+1}+k\right)}$, where $k=1,2,3, \cdots$. We define $Y_{n+1}$ to be the result of applying this construction to each segment $F$ of $Y_{n} \cap\left(\hat{E}_{n+1}-\hat{E}_{n}\right)$.

To complete the construction we must verify the three induction hypotheses. The first is trivial, and the second follows from

$$
\begin{aligned}
L\left(\partial Y_{n+1}\right) & \leqq L\left(\partial Y_{n}\right)+\pi L\left(Y_{n} \cap\left(\hat{E}_{n+1}-\hat{E}_{n}\right)\right) \\
& \leqq 4 \pi\left(1-2^{-n}\right)+\pi \cdot 2^{n} \cdot 4^{-n} \\
& <4 \pi\left(1-2^{-(n+1)}\right) .
\end{aligned}
$$

The third hypothesis must be considered in three parts:

(a) If $m=m_{1}, m_{2}, \cdots, m_{n}$, and $z \in \hat{E}_{n+1} \cap_{m} Y_{n+1}$, then $z \in \hat{E}_{n+1} \cap_{m} Y_{n}$, and the second condition in the construction of $E_{n+1}$ then implies

$$
d\left(z, \partial_{m} Y_{n+1}\right) \leqq d\left(z, \partial_{m} Y_{n}\right) \leqq\left(1-2^{-(n+1)}\right) 3^{1-m} .
$$

(b) If $m \geqq m_{n+1}$, and $z \in \hat{E}_{n} \cap_{m} Y_{n+1}$, then $z \in \hat{E}_{n} \cap_{m} Y_{n}$, and by the induction hypothesis,

$$
d\left(z, \partial_{m} Y_{n+1}\right) \leqq d\left(z, \partial_{m} Y_{n}\right) \leqq\left(1-2^{-n}\right) 3^{1-m}<\left(1-2^{-(n+1)}\right) 3^{1-m} .
$$

(c) If $m \geqq m_{n+1}$, and $z \in\left(\left(\hat{E}_{n+1}-\hat{E}_{n}\right) \cap_{m} Y_{n+1}\right)$, then the construction of $Y_{n+1}$, together with the fact that $3^{-m_{n+1}}<\rho_{n}$ implies

$$
d\left(z, \partial_{m} Y_{n+1}\right) \leqq 3^{-m}<\left(1-2^{-(n+1)}\right) 3^{1-m} .
$$

These three parts, taken together, verify the third induction hypothesis. We have therefore completed the inductive construction of the sets $\left\{E_{n}\right\}_{1}^{\infty}$ and $\left\{Y_{n}\right\}_{1}^{\infty}$, and the related integers $\left\{m_{n}\right\}_{1}^{\infty}$.

Now let $X$ be any subset of $Y=\bigcap_{n=1}^{\infty} Y_{n}$ formed by deleting from the interior of $Y$ a sequence of open discs with mutually nonintersecting boundaries of finite total length. We denote by $X_{k}$ the set formed from $X$ by filling in all holes of radius less than $3^{-k}$. Finally, let $E=\cup_{n=1}^{\infty} E_{n}, \hat{E}=\bigcup_{n=1}^{\infty} \hat{E}_{n}, K=\bar{E}$, and $\hat{K}=(\hat{E})^{-} . K$ is a Cantor set on $[-1,1]$, and if $g$ denotes the Cantor function on $K$, we define on the closed unit disc $D$ a function $f$ by $f(x+i y)=g(x)$. Then $f \in C(D)$, and is analytic (because it is locally constant) at every point of $D$ except those belonging to $\hat{K}$. 
Fix some integer $k$ : we will consider $f$ as a function on $X_{m_{k}}$. If $z \in \hat{E} \cap X_{m_{k}}$, then there exists $n \geqq k$ such that $z \in \hat{E}_{n}$. Since $X_{m_{k}} \subset_{m_{k}} Y_{n}$, $z \in \hat{E}_{n} \cap_{m_{k}} Y_{n}$; therefore we can apply the third induction hypothesis to conclude

$$
d\left(z, \partial X_{m_{k}}\right) \leqq d\left(z, \partial_{m_{k}} Y_{n}\right) \leqq\left(1-2^{-n}\right) 3^{1-m_{k}}<3^{1-m_{k}} .
$$

Therefore no point in $X_{m_{k}}$ at which $f$ is nonanalytic (i.e., no point of $\left.\hat{K} \cap X_{m_{k}}\right)$ is separated from $\partial X_{m_{k}}$ by a distance exceeding $3^{1-m_{k}}$. Since the smallest disc in the complement of $X_{m_{k}}$ has a radius of at least $3^{-m_{k}}$, we can use Mergelyan's lemma to find a rational function $r_{k}$ with poles off $X_{m_{k}}$ such that

$$
\left\|f-r_{k}\right\|_{X_{m_{k}}} \leqq c \cdot \omega\left(f ; 3^{1-m_{k}}\right) 3^{2} .
$$

Thus

$$
\operatorname{Lim}_{k \rightarrow \infty}\left\|f-r_{k}\right\|_{x}=0
$$

and this means that $R(X)$ contains the nonconstant real-valued function $f$.

\section{REFERENCE}

1. S. N. Mergelyan, Uniform approximations to functions of a complex variable, Amer. Math. Soc. Transl. No. 101 (1954), 99 pp.

St. Olaf College 